\title{
Bowman's layer encystment in cases of persistent Acanthamoeba keratitis
}

This article was published in the following Dove Press journal:

Clinical Ophthalmology

I August 2012

Number of times this article has been viewed

\section{Hideaki Yokogawa' \\ Akira Kobayashi' \\ Natsuko Yamazaki' \\ Yasuhisa Ishibashi ${ }^{2}$ \\ Yosaburo Oikawa ${ }^{3}$ \\ Masaharu Tokoro ${ }^{4}$ \\ Kazuhisa Sugiyama' \\ 'Department of Ophthalmology, Kanazawa University Graduate \\ School of Medical Science, Kanazawa, ${ }^{2}$ Department of Ophthalmology, \\ East Washinomiya Hospital, Kuki, ${ }^{3}$ Department of Medical Zoology, Kanazawa Medical University, \\ Kahoku, ${ }^{4}$ Department of Parasitology, Kanazawa University Graduate School of Medical Science, Kanazawa, Japan}

Correspondence: Akira Kobayashi Department of Ophthalmology, Kanazawa University Graduate School of Medical Science, I3-I Takara-machi, Kanazawa-shi, Ishikawa-ken 920-864I, Japan

Tel +81762652403

Fax+8I762229660

Email kobaya@kenroku.kanazawa-u.ac.jp
Background: The purpose of this study was to report Acanthamoeba encystment in Bowman's layer in Japanese cases of persistent Acanthamoeba keratitis (AK).

Methods: Laser confocal microscopic images of the cornea were obtained in vivo from 18 consecutive eyes from 17 confirmed AK patients. Retrospectively, 14 cases treated over 4 months were categorized as a nonpersistent group and three cases that required prolonged therapy for more than 6 months were categorized as a persistent group. Clinical outcomes based on final best-corrected visual acuity were retrospectively analyzed, and selected confocal images were evaluated qualitatively for abnormal findings.

Results: The final best-corrected visual acuity was significantly lower $(P<0.01)$ for patients in the persistent group compared with that in the nonpersistent group. At the initial visit, in vivo confocal microscopy demonstrated Acanthamoeba cysts exclusively in the epithelial layer in both the nonpersistent group (80\%) and the persistent group (100\%). At a subsequent follow-up visit, numerous Acanthamoeba cysts were observed in the epithelial cell layer and in Bowman's layer in all patients with persistent AK, but Acanthamoeba cysts were undetectable in all cases with nonpersistent AK tested.

Conclusion: Invasion of cysts into Bowman's layer was characteristically observed in patients with persistence of AK. This finding suggests that invasion of Acanthamoeba cysts into Bowman's layer may be a useful predictor for a persistent clinical course.

Keywords: Acanthamoeba keratitis, Bowman's layer, encystment

\section{Introduction}

Acanthamoeba keratitis (AK) is an uncommon corneal infection, and was first reported in 1974. ${ }^{1}$ The first Japanese case with AK was reported in 1988. ${ }^{2}$ AK has been strongly linked to wearing of contact lenses. ${ }^{3}$ Clinical diagnosis and management of $\mathrm{AK}$ is sometimes difficult, and patients are often misdiagnosed as having herpetic keratitis. ${ }^{3}$ Although definitive diagnosis of AK is made by biopsy or culture, in vivo confocal microscopy is helpful for diagnosis. ${ }^{4-12}$ Previously, we reported highly reflective 10-20 $\mu \mathrm{m}$ diameter Acanthamoeba cysts in the epithelium of early-stage AK using in vivo laser confocal microscopy (Heidelberg Retina Tomograph 2 Rostock Cornea Module [HRT 2-RCM], Heidelberg Engineering GmbH, Dossenheim, Germany). ${ }^{11}$ Persistent ulceration, severe inflammation, and persistent infection are clinical problems, ${ }^{13}$ and persistent amoebic antigen can induce corneal and scleral inflammation. ${ }^{13}$ In the current study, we investigated HRT 2-RCM findings for persistent $\mathrm{AK}$ and compared them with those for nonpersistent AK. 


\section{Materials and methods}

The present study was approved by the Kanazawa University Graduate School of Medical Science ethics committee, and followed the tenets of the Declaration of Helsinki. Eighteen consecutive eyes of 17 patients (nine men and eight women, of mean age $24.8 \pm 8.2$ years) with $\mathrm{AK}$ were included in this retrospective case series. In all cases, the initial clinical diagnosis was made by Parker ink-potassium hydroxide stain of an epithelial scraping and culture. Staging of AK was confirmed by Ishibashi's classification criteria. ${ }^{14}$ Pseudodendrites of the epithelium, tiny white infiltrates, and radial keratoneuritis are seen in the early stages, a ring infiltrate is seen in the transition stage, and corneal disciform structures are seen in the late stage. All patients were initially treated with a topical antifungal agent (voriconazole $1 \%$ or micafungin $0.05 \%$ ), topical chlorhexidine $0.02 \%-0.05 \%$, and epithelial debridement, with or without oral itraconazole $100 \mathrm{mg}$ per day. The need for epithelial debridement was determined by negative scraping, culture, or confocal imaging.

Three cases with persistent $\mathrm{AK}$ required prolonged therapy for more than 6 (mean $8.3 \pm 0.6$ ) months, and we retrospectively categorized these cases as a persistent group. The remaining 14 cases treated within 4 (mean $2.6 \pm 1.0$ ) months were categorized as a nonpersistent group. Clinical factors and outcomes, such as contact lens wear, topical steroid use before presentation, time interval between symptom onset and the initial visit, initial best-corrected visual acuity (BCVA), final BCVA, need for epithelial debridement, and HRT 2-RCM images between the two groups were analyzed retrospectively in order to determine the manifestations of persistent AK. HRT 2-RCM images were evaluated qualitatively for corneal structural changes or Acanthamoeba cysts, with special attention to Bowman's layer. All data analysis (unpaired $t$-test, Fisher's Exact test, and Chi-square test) was performed using SPSS Statistics software version 14.0 (SPSS Inc, Chicago, IL). $P<0.05$ was considered to be statistically significant.

\section{Results}

Table 1 shows the demographic data for the two groups. All patients in both groups were contact lens wearers. Topical steroid use before presentation and the interval between symptom onset and the initial visit were not different between the two groups. Final BCVA was significantly lower for patients in the persistent group than in the nonpersistent group $(P<0.01)$. Further, patients in the persistent group required more epithelial debridement (mean $16.3 \pm 3.2$ times) than did the nonpersistent group (mean $2.3 \pm 1.5$ times), because the persistent group required a longer period of treatment.

Table 2 shows Acanthamoeba cysts detected by HRT 2-RCM. Acanthamoeba in the epithelial layer cysts were detected in 12/14 cases (85.7\%) in the nonpersistent group, and $2 / 2$ cases $(100 \%$, data not available in case 17$)$ in the persistent group at the initial visit. Bowman's layer and the stroma were intact in both cases. No Acanthamoeba trophozoites were detected in any patients in either group.

Acanthamoeba cysts disappeared in all cases from the nonpersistent group after treatment (data missing for case 14).

Table I Demographic data for patients with persistent and nonpersistent Acanthamoeba keratitis

\begin{tabular}{|c|c|c|c|}
\hline & $\begin{array}{l}\text { Nonpersistent group } \\
n=14\end{array}$ & $\begin{array}{l}\text { Persistent group } \\
n=3\end{array}$ & $P$ value \\
\hline Age (years) & $22.5 \pm 4.5$ & $35.0 \pm 14.8$ & $0.28 *$ \\
\hline Male:female & $8: 6$ & $\mathrm{I}: 2$ & $0.45^{* *}$ \\
\hline Unilateral:bilateral & $13: 1$ & $3: 0$ & $0.82^{* *}$ \\
\hline Contact lens wearer & $14(100 \%)$ & $3(100 \%)$ & $1.00 * *$ \\
\hline (SCL:RGPCL) & $(14: 0)$ & $(2: 1)$ & \\
\hline Topical steroid use before visiting & $5(35.7 \%)$ & $2(66.7 \%)$ & $0.36 * *$ \\
\hline ( $0.1 \%$ betamethasone: $0.1 \%$ & $(1: 4: 0)$ & $(I: 0: I)$ & \\
\hline \multicolumn{4}{|l|}{ fluorometholone: $0.02 \%$ fluorometholone) } \\
\hline Interval between symptom onset and initial visit (days) & $13.1 \pm 9.4$ & $16.0 \pm 10.4$ & $0.69 *$ \\
\hline Stage at initial visit by slit-lamp biomicroscopy & II:3:0 & 2:1:0 & 0.66 *** \\
\hline \multicolumn{4}{|l|}{ initial stagetransitional stage:late stage } \\
\hline Initial BCVA $\geq 20 / 32$ & $4(28.6 \%)$ & I (33.3\%) & $0.8 I^{* *}$ \\
\hline Final $B C V A \geq 20 / 32$ & $13(92.9 \%)$ & $0(0 \%)$ & $<0.01 * *$ \\
\hline Duration of the treatment (months) & $2.6 \pm 1.0$ & $8.3 \pm 0.6$ & $<0.01 *$ \\
\hline Times of epithelial debridement & $2.3 \pm 1.5$ & $16.3 \pm 3.2$ & $0.01 *$ \\
\hline
\end{tabular}

Note: *Unpaired $t$-test; **Fisher's Exact test; ***Chi-square test.

Abbreviations: BCVA, best corrected visual acuity; SCL, soft contact lens; RGPCL, rigid gas-permeable contact lens. 
Table 2 Detection of Acanthamoeba cysts by in vivo laser confocal microscopy

\begin{tabular}{|c|c|c|c|c|}
\hline \multirow[t]{3}{*}{ Case (group) } & \multirow{3}{*}{$\begin{array}{l}\text { Age (years)/ } \\
\text { gender }\end{array}$} & \multirow{3}{*}{$\begin{array}{l}\text { Slit-lamp staging } \\
\text { at initial visit }\end{array}$} & \multicolumn{2}{|c|}{ Detection of Acanthamoeba cysts by confocal microscopy } \\
\hline & & & Initial visit & Follow-up \\
\hline & & & $\begin{array}{l}\text { Epithelium/ } \\
\text { Bowman's layer/stroma }\end{array}$ & $\begin{array}{l}\text { Epithelium/Bowman's layerl } \\
\text { stroma (day from initial visit) }\end{array}$ \\
\hline I (nonpersistent group) & $20 / M$ & Initial stage & $+1-1-$ & $-1-1-(4)$ \\
\hline 2 (nonpersistent group) & $27 / M$ & Initial stage & $+1-1-$ & $-1-1-(5)$ \\
\hline 3 (nonpersistent group) & $20 / F$ & $\begin{array}{l}\text { Initial stage } \\
\text { (bilateral) }\end{array}$ & $+/-I-(\mathrm{OU})$ & $-I-I-(I 0)(\mathrm{OU})$ \\
\hline 4 (nonpersistent group) & $18 / M$ & Initial stage & $+1-1-$ & $-1-1-(14)$ \\
\hline 5 (nonpersistent group) & $24 / \mathrm{F}$ & Initial stage & $+1-1-$ & $-1-1-(16)$ \\
\hline 6 (nonpersistent group) & I9/M & Initial stage & $+1-1-$ & $-1-1-(22)$ \\
\hline 7 (nonpersistent group) & I9/M & transient stage & $+/-1-$ & $-1-1-(30)$ \\
\hline 8 (nonpersistent group) & $31 / F$ & transient stage & $+1-1-$ & $-1-1-(30)$ \\
\hline 9 (nonpersistent group) & $19 / F$ & Initial stage & $+1-1-$ & $-1-1-(30)$ \\
\hline 10 (nonpersistent group) & $21 / F$ & Initial stage & $+1-1-$ & $-1-1-(30)$ \\
\hline II (nonpersistent group) & $23 / M$ & Initial stage & $+1-1-$ & $-1-1-(30)$ \\
\hline 12 (nonpersistent group) & $32 / M$ & Initial stage & $-1-1-$ & $-1-1-(6)$ \\
\hline 13 (nonpersistent group) & $23 / M$ & transient stage & $-1-1-$ & $-1-1-(15)$ \\
\hline 14 (nonpersistent group) & $20 / F$ & Initial stage & $-1-1-$ & Data not available \\
\hline 15 (persistent group) & $28 / F$ & Initial stage & $+1-1-$ & $+/+/-(30)$ \\
\hline 16 (persistent group) & $25 / F$ & Initial stage & $+1-1-$ & $+/+/-(50)$ \\
\hline 17 (persistent group) & $52 / M$ & transient stage & Data not available & $+/+/-(2 I)$ \\
\hline
\end{tabular}

Note: + (positive): In each layer, there is one frame $(400 \times 400 \mu \mathrm{m})$ in which more than five Acanthamoeba cysts are detectable.

Abbreviation: $\mathrm{OU}$, oculus utergue (bilateral eyes).

In contrast, numerous Acanthamoeba cysts continued to be seen in the epithelial cell layer in all cases in the persistent group (cases 15-17). Most notably, Acanthamoeba cysts forming characteristic clusters were found in Bowman's layer in all persistent cases.

\section{Case reports Case I 5 (persistent AK)}

In September 2009, a healthy 28-year-old woman presented by referral for a right keratitis refractory to 10 days of treatment with antibacterial and steroid (fluorometholone $0.02 \%$ ) eye drops. She was a wearer of frequent-replacement soft contact lenses. BCVA was 20/400 in her right eye and 20/20 in her left eye. Clinical diagnosis of earlystage AK was made by conjunctival injection, radial keratoneuritis, and subepithelial infiltration observed on slit-lamp biomicroscopy (Figure 1A). At the same time, many Acanthamoeba cysts were identified in the epithelial cell layer by HRT 2-RCM. Both direct examination and culture of the epithelium were positive for Acanthamoeba. She was treated with oral itraconazole $100 \mathrm{mg}$, topical voriconazole $1 \% 10$ times a day, topical atropine $1 \%$ twice a day, and topical chlorhexidine $0.02 \% 10$ times a day, as well as epithelial debridement. After one week of treatment, epithelium covered the total surface, and the conjunctival injection was decreased.
In October 2009, conjunctival injection and anterior chamber reaction increased despite continued application of eye drops. Because a direct examination was negative, and Acanthamoeba cysts were misidentified with leukocytes by HRT 2-RCM, low-dose topical corticosteroid (fluorometholone $0.1 \% 4$ times a day) was started on suspicion of residual noninfectious corneal inflammation and/or a drug toxicity reaction. However, deterioration of the conjunctival injection and corneal stromal cloudiness were observed (Figure 1B). Therefore, persistent infection was suspected and confirmed by in vivo laser confocal microscopy. Acanthamoeba cysts were detected in the epithelial cell layer (Figure 1C). Additionally, many Acanthamoeba cysts in the Bowman's layer were observed (Figure 1E and F). It should be noted that the diameter of the cysts in the Bowman's layer (approximately $10 \mu \mathrm{m}$ ) was smaller than in cysts observed in the epithelial cell layer $(10-15 \mu \mathrm{m})$. In the basal epithelial cell layer, numerous dendritic cells (putative Langerhans cells) with a decrease of subbasal nerves were observed (Figure 1D). An increase in background intensity of the stroma with honeycomb formation of activated keratocytes was observed (Figure 1G). However, invasion of Acanthamoeba cysts at the stromal layer was not apparent. Clusters of leukocytes were observed at the depth of the endothelial cell layer (Figure 1H). The AK was cured with slight stromal scar formation (Figure 1I). 

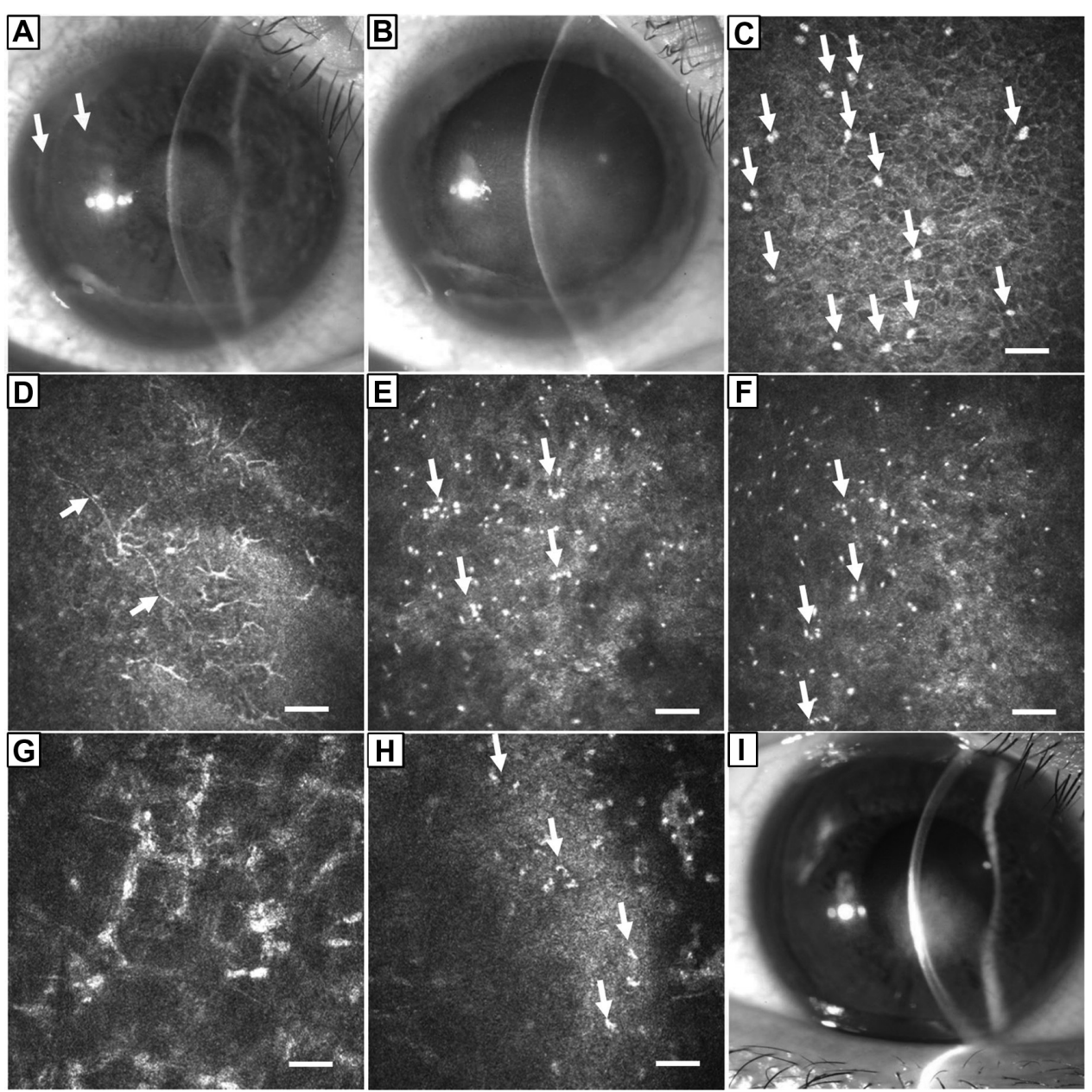

Figure I Slit lamp and in vivo laser confocal microscopic examination of case 15. (A) The right cornea on initial presentation to our hospital. Epithelial defects and subepithelial opacities were observed in the central cornea by slit-lamp biomicroscopy. Radial keratoneuritis lesions (arrows) and inflamed conjunctiva were also observed. (B) Recurrence of AK one month after treatment. Subepithelial opacity and anterior chamber reaction were increased. (C) In vivo laser confocal microscopy of recurrent AK showed a number of highly reflective, high-contrast round-shaped particles 10-15 $\mu \mathrm{m}$ in diameter (arrows), suggestive of Acanthamoeba cysts in the epithelial basal cell layer (bar $50 \mu \mathrm{m}$ ). Double-walled cysts were not detectable. (D) In the epithelial basal cell layer, numerous dendritic cells (putative Langerhans cells) were observed. A decrease in subbasal nerves (arrows) was also noted. (E and F) At the Bowman's layer level, numerous Acanthamoeba cysts were observed as highly reflective, high-contrast particles with an approximate diameter of $10 \mu \mathrm{m}$. Some of the Acanthamoeba cysts were present in small clusters (arrows). Note the diameter of the cysts in Bowman's layer ( $10 \mu \mathrm{m}$ ) was smaller than that observed in the epithelial cell layer $(10-15 \mu \mathrm{m})$. (G) Activated keratocytes forming a honeycomb pattern were observed in the stromal layer. However, Acanthamoeba cysts were not detectable in the deep stroma in this case. (H) Clusters of leukocytes (arrows) were observed in the endothelial layer. (I) Slit-lamp photograph 9 months after treatment.

Notes: The AK healed after 13 epithelial debridements with topical micafungin $0.1 \%$ and topical chlorhexidine $0.05 \%$. Despite slight stromal scar formation, the best corrected visual acuity recovered to 20/32 OD.

Abbreviations: AK, Acanthamoeba keratitis; OD, oculus dexter (right eye).

\section{Case 16 (persistent AK)}

In September 2006, a healthy 25-year-old woman presented by referral for a left keratitis refractory to 4 weeks of treatment with topical antibiotics and acyclovir ointment. She was a wearer of frequent-replacement soft contact lenses for myopia. BCVA was 20/18 in her right eye and 20/22 in her left eye. Clinical diagnosis of early-stage AK was made by conjunctival injection, radial keratoneuritis, and subepithelial infiltration observed on slit-lamp biomicroscopy (Figure 2A). At the same time, many Acanthamoeba cysts were identified in the epithelial cell layer by HRT 2-RCM.
Direct examination was positive but culture was negative for Acanthamoeba. She was treated with oral itraconazole $100 \mathrm{mg}$, topical micafungin $0.05 \% 10$ times a day, and topical chlorhexidine $0.05 \% 10$ times a day, as well as epithelial debridement.

In October 2006, the epithelium covered the total surface and conjunctival injection was decreased. In November 2006, an epithelial defect appeared. Because an epithelial scraping was negative for Acanthamoeba cysts, a low-dose topical corticosteroid (fluorometholone $0.1 \%$ four times a day) was started on suspicion of residual noninfectious 


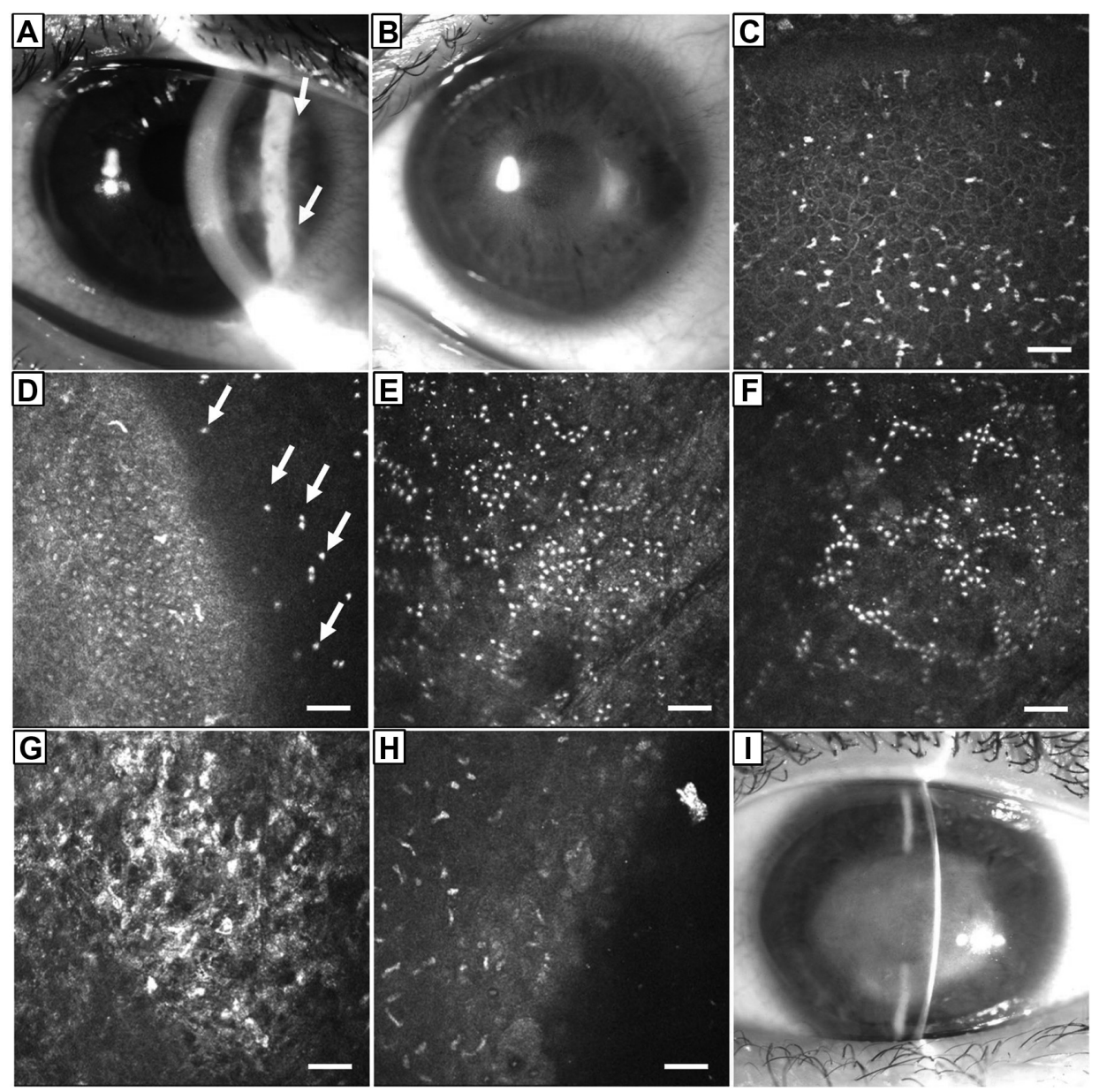

Figure 2 Slit-lamp and in vivo laser confocal microscopic examination of case 16. (A) The left cornea at initial presentation to our hospital. Subepithelial opacities and radial keratoneuritis lesions (arrows) were observed by slit-lamp biomicroscopy. Inflamed conjunctiva was also observed. (B) AK recurred 50 days after treatment. Ring-form infiltration appeared. Anterior chamber reaction increased. (C) In vivo laser confocal microscopy of recurrent AK showed a number of highly reflective, high-contrast particles 10-15 $\mu \mathrm{m}$ in diameter, suggesting Acanthamoeba cysts in the epithelial basal cell layer (bar $50 \mu \mathrm{m}$ ). (D) Oblique view of the superficial cornea. Acanthamoeba cysts (arrows) were clearly recognized in Bowman's layer. (E and F) In Bowman's layer, characteristic clusters of Acanthamoeba cysts were observed as highly reflective, highcontrast particles with a diameter of approximately $10 \mu \mathrm{m}$. (G) The superficial stroma showed high reflectivity without Acanthamoeba cysts. (H) In the endothelial layer, leukocytes were observed. (I) Slit-lamp photograph 12 months after treatment.

Notes: The AK healed after 20 epithelial debridements with topical micafungin $0.1 \%$ and topical chlorhexidine $0.05 \%$. Despite stromal scar formation, the best corrected visual acuity had recovered to $20 / 60$ OS.

Abbreviations: AK, Acanthamoeba keratitis; OS, oculus sinister (left eye).

corneal inflammation and/or drug toxicity reaction without confocal image data. However, radial keratoneuritis ring infiltration appeared (Figure 2B). Therefore, persistent infection was suspected and confirmed by in vivo laser confocal microscopy. Acanthamoeba cysts were detected in the epithelial cell layer (Figure 2C). Additionally, many Acanthamoeba cysts in the Bowman's layer were observed (Figure 2D-F). It should be noted that the diameter of cysts in Bowman's layer (approximately $10 \mu \mathrm{m}$ ) was smaller than in those observed in the epithelial cell layer $(10-15 \mu \mathrm{m})$. An increase in background intensity of the stroma with honeycomb formation of activated keratocytes was observed (Figure 2G). However, invasion of Acanthamoeba cysts into the stromal layer was not apparent. Clusters of leukocytes were observed at the depth of the endothelial cell layer (Figure $2 \mathrm{H}$ ). Finally, the AK was cured, with dense stromal scar formation (Figure 2I).

\section{Discussion}

We have previously reported the usefulness of in vivo laser confocal microscopy for detection of Acanthamoeba cysts in the epithelial cell layer during early-stage AK. ${ }^{11}$ In the present study, we demonstrated the characteristics of both nonpersistent and persistent $\mathrm{AK}$ in vivo using laser confocal microscopy. Acanthamoeba cysts were limited to the epithelial cell layer in $12 / 14$ cases (85.7\%) in nonpersistent patients, and in $2 / 2$ cases in persistent patients at the visit. Most notably, numerous Acanthamoeba cysts forming 
clusters in the Bowman's layer were characteristically observed in all cases of persistent $\mathrm{AK}$ but not in nonpersistent $\mathrm{AK}$ at subsequent follow-up visits. This finding indicates that invasion of Acanthamoeba cysts into Bowman's layer observed with confocal microscopy is a characteristic sign of persistent AK.

As previously described, cultured Acanthamoeba cysts formed clusters in the medium and were relatively larger in size (10-20 $\mu \mathrm{m}$ in diameter). ${ }^{11}$ The Acanthamoeba cysts appeared as highly reflective, round-shaped, or stellate-shaped particles in culture. In contrast, the shapes of Acanthamoeba cysts in the corneal epithelium tended to be rounder and smaller in size. Therefore, we hypothesized that epithelial cells might compress Acanthamoeba cysts in vivo, which does not occur in the culture enviroment. ${ }^{11}$ In other words, Acanthamoeba cysts show a wider range of shapes when in culture medium. Interestingly, as observed in this study, the size of the Acanthamoeba cysts is more compact in Bowman's layer than in the epithelial cell layer. Bowman's layer probably serves as a mechanical barrier to Acanthamoeba cysts/ trophozoites, and the infection only advances when the cysts/ trophozoites destroyed the barrier. In fact, amoebic invasion into the corneal stroma is thought to be a bad prognostic factor. ${ }^{15}$ In this study, we were not able to find an association between persistence and epidemiological factors, such as contact lens wear, topical steroid use before presentation, and interval between symptom onset and initial visit.

Our 14 cases (15 eyes) of nonpersistent AK healed with good final BCVA within only 4 months, indicating that AK is curable when Acanthamoeba cysts are found to be localized within the epithelium on confocal microscopy. However, the three cases of persistent AK were prolonged (8-9 months) despite intensive therapy consisting of multiple epithelial debridements, antifungal agents, and topical chlorhexidine. It was difficult to diagnose persistent $\mathrm{AK}$ infection in cases 15 and 16 , since epithelial scrapings were negative. This falsenegative information led us to suspect residual noninfectious corneal inflammation and/or a drug toxicity reaction, resulting in use of low-dose topical corticosteroid eye drops. We learned from these cases that we should be more careful before using steroid eye drops for eyes with AK. Polymerase chain reaction investigation of the genome in the epithelium could be a novel sensitive diagnostic tool, but is not always available. Repeated confocal microscopic examinations over short intervals may be useful to monitor the recurrence of AK before steroid eye drops are initiated to treat residual inflammation and/or drug toxicity. However, confocal microscopy images of the epithelium were quite confusing at this time and highly reflective particles were confused with Acanthamoeba cysts and leukocytes. Therefore, in such cases, maximum attention should be focused on Bowman's layer to see if there are characteristic images of numerous clusters of Acanthamoeba cysts as shown in this study. Acanthamoeba cysts could be distinguished from leukocytes by confocal findings such as cluster formation and characteristic round shape, whereas leukocytes tend to have a lobular nucleus.

In conclusion, in vivo laser confocal microscopy was proven to be useful for the diagnosis of AK by demonstrating Acanthamoeba cysts in the epithelium. With persistence of AK, invasion of cysts into Bowman's layer forming clusters were characteristically observed. This finding suggests that invasion of Acanthamoeba cysts into Bowman's layer may be a useful predictor of a persistent clinical course. Further biomechanical and anatomic studies in a larger number of patients are needed to reveal the pathophysiology of persistent $\mathrm{AK}$.

\section{Disclosure}

The authors report no conflicts of interest in this work.

\section{References}

1. Nagington J, Watson PG, Playfair TJ, McGill J, Jones BR, Steele AD. Amoebic infection of the eye. Lancet. 1974;2(7896):1537-1540.

2. Ishibashi Y, Matsumoto Y, Watanabe R, et al. A case of Acanthamoeba keratitis: the first case report in Japan. J Jpn Ophthalmol Soc. 1988; 92(6):963-972.

3. Hammersmith KM. Diagnosis and management of Acanthamoeba keratitis. Curr Opin Ophthalmol. 2006;17(4):327-331.

4. Winchester K, Mathers WD, Sutphin JE, Daley TE. Diagnosis of Acanthamoeba keratitis in vivo with confocal microscopy. Cornea. 1995;14(1):10-17.

5. Cavanagh HD, Petroll WM, Alizadeh H, He YG, McCulley JP, Jester JV. Clinical and diagnostic use of in vivo confocal microscopy in patients with corneal disease. Ophthalmology. 1993;100(10):1444-1454.

6. Kaufman SC, Musch DC, Belin MW, et al. Confocal microscopy: a report by the American Academy of Ophthalmology. Ophthalmology. 2004;111(2):396-406.

7. Nakano E, Oliveira M, Portellinha W, de Freitas D, Nakano K. Confocal microscopy in early diagnosis of Acanthamoeba keratitis. $J$ Refract Surg. 2004;20(Suppl 5):S737-S740.

8. Parmar DN, Awwad ST, Petroll WM, Bowman RW, McCulley JP, Cavanagh HD. Tandem scanning confocal corneal microscopy in the diagnosis of suspected Acanthamoeba keratitis. Ophthalmology. 2006; 113(4):538-547.

9. Chew SJ, Beuerman RW, Assouline M, Kaufman HE, Barron BA, Hill JM. Early diagnosis of infectious keratitis with in vivo real time confocal microscopy. CLAO J. 1992;18(8):197-201.

10. Pfister DR, Cameron JD, Krachmer JH, Holland EJ. Confocal microscopy findings of Acanthamoeba keratitis. Am J Ophthalmol. 1996;121(2):119-128.

11. Kobayashi A, Ishibashi Y, Oikawa Y, Yokogawa H, Sugiyama K. In vivo and ex vivo laser confocal microscopy findings in patients with early-stage Acanthamoeba keratitis. Cornea. 2008;27(4):439-445.

12. Shiraishi A, Uno T, Oka N, Hara Y, Yamaguchi M, Ohashi Y. In vivo and in vitro laser confocal microscopy to diagnose Acanthamoeba keratitis. Cornea. 2010;29(8):861-865. 
13. Yang YF, Matheson M, Dart JK, Cree IA. Persistence of acanthamoeba antigen following Acanthamoeba keratitis. Br J Ophthalmol. 2001; 85(3):277-280.

14. IshibashiY.Acanthamoeba keratitis. Ophthalmologica. 1997;211 Suppl 1: 39-44.
15. Chew HF, Yildiz EH, Hammersmith KM, et al. Clinical outcomes and prognostic factors associated with Acanthamoeba keratitis. Cornea. 2011;30(4):435-441.

\section{Publish your work in this journal}

Clinical Ophthalmology is an international, peer-reviewed journal covering all subspecialties within ophthalmology. Key topics include: Optometry; Visual science; Pharmacology and drug therapy in eye diseases; Basic Sciences; Primary and Secondary eye care; Patien Safety and Quality of Care Improvements. This journal is indexed on Submit your manuscript here: http://www.dovepress.com/clinical-ophthalmology-journal

\section{Dovepress}

PubMed Central and CAS, and is the official journal of The Society of Clinical Ophthalmology (SCO). The manuscript management system is completely online and includes a very quick and fair peer-review system, which is all easy to use. Visit http://www.dovepress.com/ testimonials.php to read real quotes from published authors. 